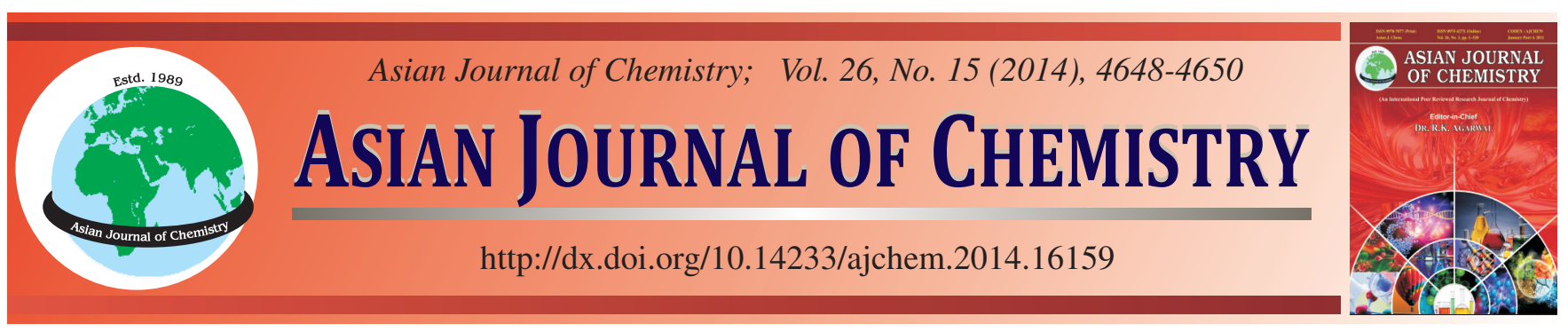

\title{
Total Phenolic, Total Flavonoid and Antioxidant Activity of Euonymus fortunei by HPLC-Based Analysis
}

\section{XI-Lin OuYang ${ }^{1,2}$, XIAN-Ming FAng $^{3}$, Heng-Shan WAnG $^{2, *}$, LiU-XIN WeI ${ }^{2}$ and Ying-Ming Pan ${ }^{2, *}$}

${ }^{1}$ Youjiang Medical University for Nationalities, Baise 533000, P.R. China

${ }^{2}$ State Key Laboratory Cultivation Base for the Chemistry and Molecular Engineering of Medicinal Resources, School of Chemistry \& Pharmacy of Guangxi Normal University, Guilin 541004, P.R. China

${ }^{3}$ Guangxi Traditional Chinese Medical University, Nanning 530001, P.R. China

*Corresponding authors: Tel/Fax: +86 773 2120958; E-mail: hengshan_wang@yahoo.com; panym2005@sina.com

Euonymus fortunei is one of the most important folk medicinal plants in Guangxi of China and also used as a healthy tea for enhancement of immunity and disease resistance. The ethanol crude extract and its fractions of $E$. fortune $i$ were investigated to determine their total phenolic and flavonoid contents, as well as their antioxidant activity, which assessed by 1,1-diphenyl-2-picrylhydrazyl (DPPH) and 2,2'azinobis (3-ethylbenzothiazoline-6-sulfonic acid) diamonium salt (ABTS) radical scavenging assay. Its' compositions were analyzed by HPLC. The ethyl acetate fraction possesses the high total phenolic and total flavonoid with the contents of $707.2 \mathrm{mg}$ gallic acid equivalents and $581 \mathrm{mg}$ rutin equivalents $\mathrm{g}^{-1}$ extract, respectively. The ethyl acetate fraction also exhibited the strongest activity with $\mathrm{EC}_{50}$ value of $0.25 \mathrm{mg} / \mathrm{mL}$, followed by the $n$-butanol fraction, while petroleum ether and water fractions were less active than crude extract. HPLC analysis of the ethanol crude extracts indicated the presence of a large number of flavonoids and its' glycosides. E. fortunei could be used as an important natural source of antioxidants and a substantial source of healthy tea, as well as a medicinal plant.

Keywords: Euonymus fortunei, Flavonoid, Antioxidant, HPLC analysis.

\section{INTRODUCTION}

Euonymus fortunei (Turcz.) Hand.-Mazz., belonging to the genus of Euonymus, has been used in folk medicine to treat traumatic injury and improve immunity system ${ }^{1}$. E. fortunei leaves were made for a healthy tea used in some ethnic minority areas in Guangxi, China. Recent studies revealed that the extracts of E. fortunei could inhibit non-enzymatic lipid peroxidation and some phenolic compounds (polyphenols, tannins, phenolic acids and flavonoids) were isolated and identified from the cane part ${ }^{2,3}$. In our previous work, six flavonoid glycosides were isolated from the ethanol extract of $E$. fortune $i^{4}$. Two compounds, namely kaempferol-3,7- $O$ - $\alpha$-dirhamnopyranoside and kaempferol-3-(4"-O-acetyl)- $O$ - $\alpha$-L-rhamnopyranoside-7- $O-\alpha$-L-rhamnopyranoside, were proved as the major class of constituents, with high contents of 0.03-0.54 and 0.12$0.67 \mathrm{mg} / \mathrm{g}$, respectively ${ }^{5}$. Phenolic compounds are known to act as antioxidants and flavonoids are one of the most important phenolic compounds with radical scavenging properties ${ }^{6}$. These suggested that E. fortunei might have important antioxidant properties.

However, to the best of our knowledge, data on the antioxidant properties of $E$. fortunei extracts are scarce. In continuation of our previous work on E. fortunei, the objectives of this study were to quantitatively analyze total phenolic and total flavonoid contents in ethanol extract and its various fractions and then evaluate their antioxidant activity. Finally, the main chemical compositions were confirmed by HPLC analysis to explain the reasons that $E$. fortunei can be used as a healthy tea.

\section{EXPERIMENTAL}

The aerial parts of $E$. fortunei were collected from Yongfu county of Guangxi in October 2008. The plant material was dried under shade at room temperature by wind and was pulverized by a mill machine. Voucher specimen (No YF081009) was deposited at the State Key Laboratory Cultivation Base for the Chemistry and Molecular Engineering of Medicinal Resources, Guangxi Normal University, Guilin, Guangxi.

Extraction and partition: Powdered aerial parts of $E$. fortunei $(30 \mathrm{~g})$ were extracted with $300 \mathrm{~mL}$ of aqueous ethanol $(70 \%, v / v)$ by heating under reflux for $1 \mathrm{~h}$. The extract was evaporated under vacuum and a residue $(4.06 \mathrm{~g})$ was obtained. The residue was dissolved in purified water and subjected to repeated liquid-liquid extraction with solvents of ascending polarity affording known weight of four fractions. All samples were stored at $-4{ }^{\circ} \mathrm{C}$ until analyzed. 
Total phenolic content and total flavonoid content: Total phenolic content was determined according to the FolinCiocalteu method ${ }^{7}$. Gallic acid solutions $(0.5 \mathrm{~mL})$ in the concentration range $0.02-0.05 \mathrm{mg} / \mathrm{mL}$ were used to prepare a calibration curve. The concentration of total phenolic compounds was determined as micrograms of gallic acid equivalents per gram dry weight of extracts using the linear equation:

Absorbance $=0.1317+28.03 \times$ gallic acid $(\mathrm{mg} / \mathrm{mL}), \mathrm{R}=$ 0.9860 .

The flavonoid content was measured using a colorimetric assay $^{8}$. Rutin solutions $(0.5 \mathrm{~mL})$ in the concentration range $0.06-0.3 \mathrm{mg} / \mathrm{mL}$ were used to prepare a calibration curve. The amount of flavonoids was calculated by the following formula: Absorbance $=3.23667 \times$ rutin $(\mathrm{mg} / \mathrm{mL})-0.0366, \mathrm{R}=0.9997$.

All samples were performed in triplicate and the results were averaged.

DPPH radical scavenging activity: The DPPH free radical scavenging activity was carried out according to the DPPH method reported in previous research ${ }^{9}$. The antioxidant activity was expressed as a percentage of scavenging activity (EC \%) on DPPH radical:

$\mathrm{EC} \%=[1-($ absorbance of sample $) /($ absorbance of control $)]$ $\times 100 \%$.

ABTS radical scavenging activity: The ABTS radical scavenging activity was determined in accordance with the method of Cai et al. ${ }^{10}$. Prior to analysis, the ABTS solution was diluted with phosphate buffer to produce a solution with an absorbance of $0.80 \pm 0.03$ at $\lambda=734 \mathrm{~nm}$. A test sample $(0.1 \mathrm{~mL})$ was mixed with $3.9 \mathrm{~mL}$ of ABTS solution thoroughly and stood for a further 3 min, after which the absorbance was measured at $734 \mathrm{~nm}$. Antioxidant activity was expressed as a percentage of scavenging activity of ABTS, calculated from the same equation as for DPPH.

HPLC analysis: Optimizations of sample extraction and chromatography conditions of HPLC analysis were carried out as reported in previous research ${ }^{5}$.

Statistical analysis: SPSS 16 for Windows (SPSS Inc., Chicago, IL, USA) was used for statistical analysis. One-way analysis of variance (ANOVA) was used to determine the differences among the means. The criterion for significance was set at $\mathrm{p}<0.05$.

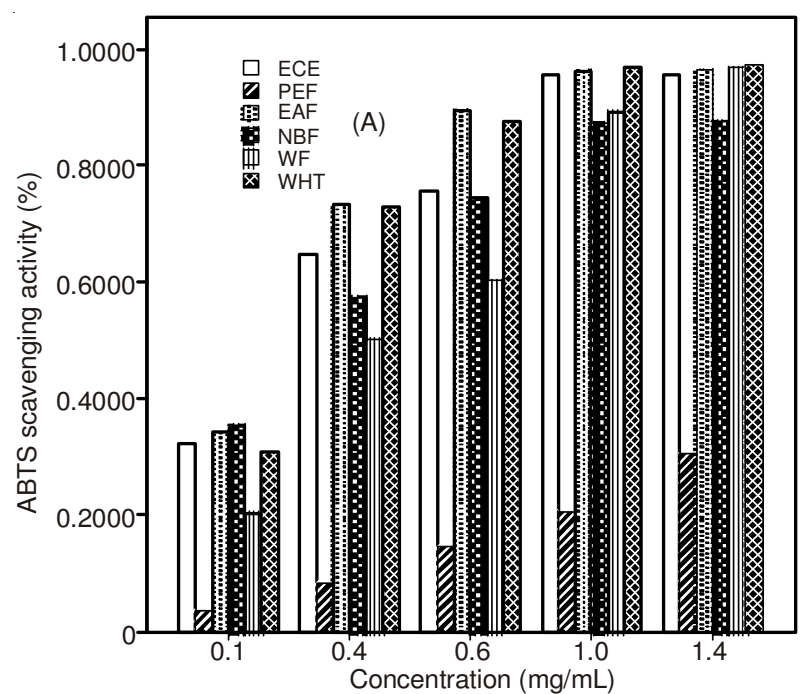

\section{RESULTS AND DISCUSSION}

Total phenolic and total flavonoid contents: As is shown in Table-1, the highest content of total phenolic was found in the ethyl acetate fraction (EAF), which was increased nearly twice comparing that of the ethanol crude extract (ECE). It possesses the total phenolic content with content of $707.2 \mathrm{mg}$ expressed as gallic acid per gram of extract, superior to that of $408.6 \mathrm{mg}$ in ECE. The total phenolic of $n$-butanol fraction $(\mathrm{NBF})$ are also shown higher content than ECE, whereas total phenolic contents of petroleum ether fraction (PEF) and water fraction (WF) are decreased compared that of ECE. Total flavonoid content followed a similar pattern as total phenolic content, but they are different in order. A significant difference was also observed in total flavonoid of EAF and WF among the fractions: flavonoid content in EAF is $581.0 \mathrm{mg}$ of rutin equivalents in per mg extract, increased more than four times comparing that of ECE, whereas in WF, it is $36.1 \mathrm{mg}$. As we all know, high phenolic content was usually correlated with high radical scavenging activity, therefore, EAF may be one of the strongest antioxidant fractions.

TABLE-1

TOTAL PHENOLIC AND TOTAL FLAVONOID CONTENTS OF ETHANOL CRUDE EXTRACT AND ITS VARIOUS FRACTIONS FROM $E$. fortunei

\begin{tabular}{lccc}
\hline & $\begin{array}{c}\text { Weight } \\
(\mathrm{mg})\end{array}$ & $\begin{array}{c}\text { Total phenolic }(\mathrm{mg} \\
\text { GAE/g extract) }^{\mathrm{b}}\end{array}$ & $\begin{array}{c}\text { Total flavonoid }(\mathrm{mg} \\
\text { RE/g extract) }\end{array}$ \\
\hline ECE & $4064.9^{\mathrm{a}}$ & 408.6 & 131.8 \\
PEF & 125.1 & 75.7 & 43.5 \\
EAF & 195.7 & 707.2 & 581.0 \\
NBF & 721.0 & 474.5 & 183.4 \\
WF & 1525.9 & 313.2 & 36.1 \\
\hline
\end{tabular}

${ }^{a}$ Data were presented as means of triplicate replication determinations ${ }^{\mathrm{b}} \mathrm{GAE}$, gallic acid equivalents, ${ }^{\mathrm{C}} \mathrm{RE}$, rutin equivalents

Analysis of antioxidant activity: In this work, DPPH and ABTS free radical scavenging assays were carried out to evaluate the antioxidant activities and the results are shown in Fig. 1. All the samples show antioxidant activity in the order of EAF $>\mathrm{NBF}>\mathrm{ECE}>\mathrm{WF}>\mathrm{PEF}$. Ethyl acetate fraction has the highest free radical scavenging activity, followed by NBF.

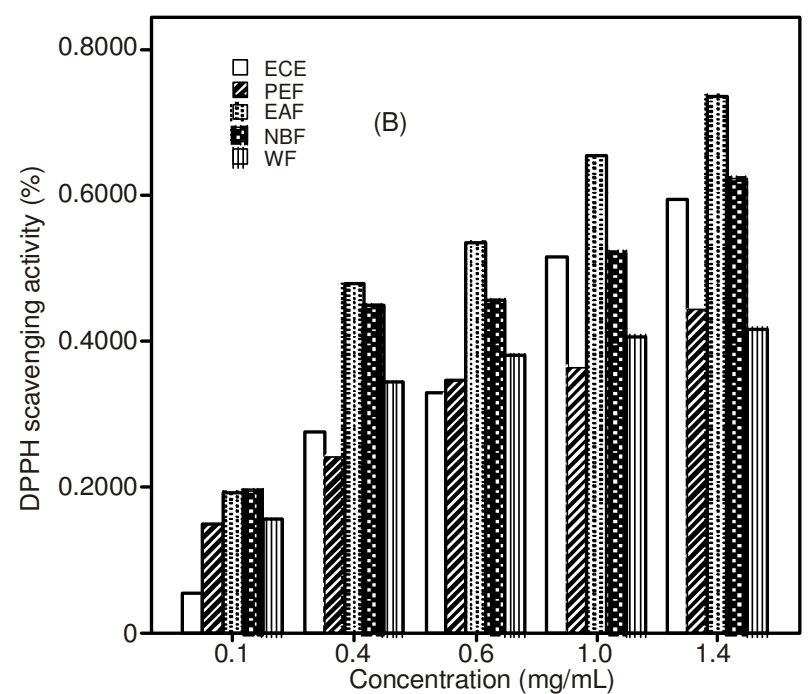

Fig. 1. DPPH and ABTS assay of various samples obtained from E. fortunei. In each concentration, all values are statistically different (p < 0.05). A, DPPH assay; B, ABTS assay 
The PEF exhibits the weakest activity, significantly different from any other fractions. According to the $\mathrm{EC}_{50}$ values (concentration of samples required to scavenge $50 \%$ of DPPH free radicals), the $\mathrm{EAF}$ show the $\mathrm{EC}_{50}$ value of $0.25 \mathrm{mg} / \mathrm{mL}$, lower than other extracts (not shown), as well as BHT, a synthetic antioxidants with $\mathrm{EC}_{50}$ value of $0.62 \mathrm{mg} / \mathrm{mL}$. On DPPH free radicals, we can see the order of $\mathrm{EC}_{50}$ values: $\mathrm{EAF}<\mathrm{NBF}<$ $\mathrm{ECE}<\mathrm{BHT} \approx \mathrm{WF}<\mathrm{PEF}$.

The ABTS radical scavenging activities of various samples are shown in Fig. 1B. All the samples also showed ABTS radical scavenging activity. Among the four fractions and crude extract of $E$. fortunei, EAF exhibited the highest activity. It has similar inhibition of ABTS at all test concentrations with BHT, under the same experimental conditions, followed by NBF and ECE. The PEF showed the weakest radical scavenging ability.

HPLC analysis: In general, the flavonoids typically yield two UV maximum absorption wavelengths: The first maximum absorption wavelength is found from $254 \mathrm{~nm}$ to $261 \mathrm{~nm}$ (benzoyl system, band I) and the second is shifted from 333 $\mathrm{nm}$ to $356 \mathrm{~nm}$ (cinnamon acyl system, band II). If the absorption of peaks contains these two maximum wavelengths, we can consider that it is flavonoid compounds ${ }^{11}$.

Based on the HPLC analysis of E. fortunei, characteristic peaks show similar at $254 \mathrm{~nm}$ and $340 \mathrm{~nm}$ after $15 \mathrm{~min}$, while major peaks at $254 \mathrm{~nm}$ are quite different with that at $340 \mathrm{~nm}$ in the first $15 \mathrm{~min}$ (Fig. 2). At the first $15 \mathrm{~min}, n$-butanol and water soluble fraction, contained flavonoids and other nonflavonoids, were eluted first. The ethyl acetate extract is mainly allotted between 15-30 min and its chemical composition contains more than 35 compounds of flavonoids. This result indicates flavonoid compounds are the major constituents in the ethanol crude extract of ethanol extract of E. fortunei. Thus, flavonoid compounds as the active constituents would be responsible for high antioxidant activity of this plant.

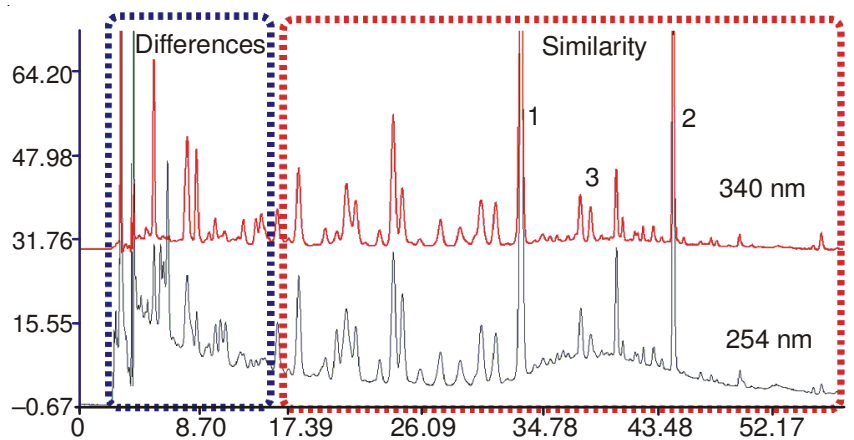

Fig. 2. Liquid chromatogram of ethanol extract at $254 \mathrm{~nm}$ and $340 \mathrm{~nm}$

\section{Conclusion}

The results of the present study show that all the samples exhibited different extent of antioxidant activity. The radical scavenging activity was increased in ethyl acetate fraction (EAF) and $n$-butanol fraction (NBF), whereas the activity of petroleum ether fraction (PEF) and water fraction (WF) were decreased compared with ethanol crude extract (ECE). In addition, EAF shows a higher potency than other fractions and the crude extract. The flavonoids constituents of E. fortunei are mainly responsible for their high antioxidant activity. The results would certainly help to ascertain the potency of the ethanol crude extract of $E$. fortunei as a potential source of natural antioxidants and can be used as a new source of nonCamellia tea.

\section{ACKNOWLEDGEMENTS}

This study is supported by 973 projects (No. 2011CB512005), Key Technology R\&D program (No. 2006BAI06A18-14), the Natural Science Foundation of Guangxi Province (No. 2012GXNSFAA053027) and the Fund of Guangxi Key Laboratory of Functional Phytochemicals Research and Utilization (Nos. FPRU2011-1 and FPRU2013-7).

\section{REFERENCES}

1. Jiangsu New Medical College, Shanghai Science and Technology Press, Shanghai, China, (1986).

2. M. Liao, Y. Yang, G. Yang and Z. Mei, J. South-Central Univ. National. (Nat. Sc. Ed.), 28, 51 (2009).

3. F.L. Qu, Q.L. Ding and H.M. Zhang, Med. J. National Defending Forces in Southwest Chin., 12, 349 (2002).

4. X.L. Ouyang, L.X. Wei, X.M. Fang, H.S. Wang and Y.M. Pan, Chem. Nat. Comp., 49, 428 (2013).

5. X.L. Ouyang, X.M. Fang, Y.M. Pan, L.X. Wei and H.S. Wang, Acta Chromatogr., 24, 301 (2012).

6. W. Kriaa, H. Fetoui, M. Makni, N. Zeghal and N.-E. Drira, Int. J. Food Prop., 15, 1220 (2012).

7. C.I.G. Tuberoso, P. Montoro, S. Piacente, G. Corona, M. Deiana, M.A. Dessì, C. Pizza and P. Cabras, J. Pharm. Biomed. Anal., 50, 440 (2009).

8. G. Miliauskas, P.R. Venskutonis and T.A. van Beek, Food Chem., 85, 231 (2004).

9. Y. Pan, K. Wang, S. Huang, H. Wang, X. Mu, C. He, X. Ji, J. Zhang and F. Huang, Food Chem., 106, 1264 (2008).

10. Y. Cai, Q. Luo, M. Sun and H. Corke, Life Sci., 74, 2157 (2004).

11. J. Xie, L. Shi, X. Zhu, P. Wang, Y. Zhao and W. Su, Innov. Food Sci. Emerg. Technol., 12, 146 (2011). 